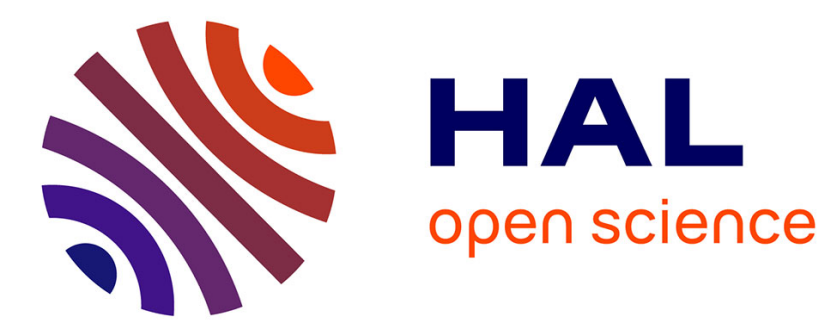

\title{
Etude du couplage thermochimique au sein de matrices cimentaires en cours d'hydratation
}

Pierre Mounanga, Philippe Poullain, Guy Bastian, Abdelhafid Khelidj

\section{To cite this version:}

Pierre Mounanga, Philippe Poullain, Guy Bastian, Abdelhafid Khelidj. Etude du couplage thermochimique au sein de matrices cimentaires en cours d'hydratation. Revue Européenne de Génie Civil, 2007, 11 (6), pp.725-737. 10.1080/17747120.2007.9692954 . hal-01006815

\section{HAL Id: hal-01006815 https://hal.science/hal-01006815}

Submitted on 12 Mar 2017

HAL is a multi-disciplinary open access archive for the deposit and dissemination of scientific research documents, whether they are published or not. The documents may come from teaching and research institutions in France or abroad, or from public or private research centers.
L'archive ouverte pluridisciplinaire HAL, est destinée au dépôt et à la diffusion de documents scientifiques de niveau recherche, publiés ou non, émanant des établissements d'enseignement et de recherche français ou étrangers, des laboratoires publics ou privés. 


\title{
Etude du couplage thermochimique au sein de matrices cimentaires en cours d'hydratation
}

\author{
Pierre Mounanga — Philippe Poullain - Guy Bastian — Abdelhafid Khelidj \\ GeM - UMR CNRS 6183, Institut de Recherche en Génie Civil et Mécanique \\ IUT de Saint-Nazaire \\ 58 Rue Michel Ange, 44606 Saint-Nazaire Cedex
}

\begin{abstract}
RÉSUMÉ. Au très jeune âge, les matrices cimentaires sont sujettes à une élévation de température liée au double caractère exothermique et thermoactivé de l'hydratation du ciment. Les paramètres du couplage thermochimique (conductivité thermique, capacité calorifique, énergie d'activation apparente et affinité chimique) caractérisant ce comportement ont été identifiés au travers de la littérature et mesurés sur pâtes de ciment pendant les premières 24 heures d'hydratation. Ils ont ensuite été utilisés dans un modèle numérique 2D permettant de simuler la cinétique d'hydratation et le champ de température de matrices cimentaires durcissantes. L'objectif visé est de mesurer l'influence du choix des paramètres thermophysiques sur la prédiction du comportement de ces matériaux au très jeune âge. L'étude de sensibilité montre que la valeur des propriétés thermiques influe significativement sur l'évolution du champ de température et très peu sur la cinétique d'hydratation.
\end{abstract}

ABSTRACT. At very early age, cementitious systems can undergo thermal variations due to the double exothermic and thermoactivated property of cement hydration. The parameters of this thermochemical coupling (thermal conductivity, specific heat capacity, apparent activation energy and chemical affinity) have been identified from the literature and measured on cement pastes during the first 24 hours of hydration. They are then used in a $2 D$ numerical model for the computing of hydration degree and temperature field of hardening cementbased materials. The aim is to quantify the effect of the thermophysical parameter values on the prediction of the material's behaviour at very early age. The sensitivity study shows that the influence of thermal property values is particularly significant in the case of temperature field computation.

MOTS-CLÉS : couplage thermochimique, pâte de ciment, hydratation, très jeune âge, modélisation.

KEYWORDS: Thermochemical coupling, cement paste, hydration, very early age, modelling. 


\section{Introduction}

La prise des matériaux à base de liant hydraulique s'accompagne d'un important dégagement de chaleur lié au caractère exothermique des réactions d'hydratation $\mathrm{du}$ ciment. Dans les pièces massives en béton (typiquement d'épaisseur supérieure à 40 centimètres - Acker, 1992), la prise se déroule «à chaud ». Le gradient thermique, qui apparaît entre le cœur et la surface de la pièce, peut être à l'origine d'une fissuration de peau. Par ailleurs, au niveau des reprises de bétonnage, la contraction thermique, engendrée par le retour à la température ambiante, est gênée par la rigidité des zones précédentes. Ce retrait empêché peut entraîner des fissurations en bloc ou des fissurations traversantes (Acker, 1988; Torrenti et al., 1995; de Schutter, 2002 ; Lackner et Mang, 2004 ; Waller et al., 2004).

L'estimation des déformations d'origine thermique des matrices cimentaires exige la connaissance de leurs propriétés de transfert thermique (conductivité thermique $\lambda$ et capacité calorifique $\rho C_{P}$ ) au très jeune âge. Par ailleurs, le double caractère thermoactivé et exothermique du processus d'hydratation lie l'évolution du champ de température à l'avancement du degré d'hydratation : la prise en compte de ce couplage thermochimique nécessite la détermination de deux paramètres supplémentaires, l'énergie d'activation apparente $E_{a}$ et l'affinité chimique $\tilde{A}$ du liant (Ulm et Coussy, 1998).

Cet article présente de nouvelles données expérimentales de $\lambda, \rho C_{P}, E_{a}$ et $\tilde{A}$ mesurées sur des pâtes de ciment de type CEM I pendant les premières 24 heures d'hydratation. Ces caractéristiques sont ensuite utilisées dans un modèle $2 \mathrm{D}$ en différences finies implémenté dans Matlab® (MathWorks inc.), simulant l'évolution des champs de degré d'hydratation $\alpha(t)$ et de température $T(t)$ de matrices cimentaires. Les simulations réalisées ont permis de quantifier la sensibilité du calcul de $\alpha(t)$ et $T(t)$ vis-à-vis des valeurs de $\lambda$ et $\rho C_{P}$ utilisées.

\section{Détermination expérimentale des paramètres du couplage thermochimique}

\subsection{Caractéristiques physico-chimiques : $E_{a}$ et $\tilde{A}$}

$E_{a}$ et $\tilde{A}$ sont, respectivement, l'énergie d'activation apparente et l'affinité chimique entre le ciment et l'eau. $E_{a}$ permet de quantifier l'influence de la température sur la cinétique d'hydratation du ciment; elle peut se calculer comme (d'Aloïa et Chanvillard, 2002 ; Wirquin et al., 2002) : 
$E_{a}(t)=\frac{R \cdot\left[\ln \left(\frac{d \alpha}{d t}\right)_{T_{r e f}}-\ln \left(\frac{d \alpha}{d t}\right)_{T}\right]}{\frac{1}{T(t)}-\frac{1}{T_{\text {ref }}}}$

[1]

où $\alpha(t)$ est le degré d'hydratation du ciment [-], T(t) la température du matériau [K], $T_{\text {ref }}$ une température de référence (293 $\mathrm{K}$, en général), $t$ le temps [s] et $R$ la constante des gaz parfaits $\left(8,314 \mathrm{~J} \mathrm{~mol}^{-1} \mathrm{~K}^{-1}\right)$.

$\tilde{A}(t)$ est définie comme le mécanisme moteur de l'hydratation (Bernard et al., 2003). Elle s'exprime sous la forme suivante (Ulm et Coussy, 1998) :

$$
\tilde{A}(t)=\frac{d \alpha}{d t} \cdot \exp \left(\frac{E_{a}}{R T}\right)
$$

Le calcul de $E_{a}$ et $\tilde{A}$, propriétés évoluant en fonction de la maturité du matériau, nécessite la mesure du degré d'hydratation. Ce dernier a été obtenu à partir d'essais de retrait chimique $\Delta V_{\text {chim }}$ mesuré par gravimétrie (Mounanga et al., 2004a) :

$$
\alpha(t)=\frac{\Delta V_{\text {chim }}(t)}{\Delta V_{\text {chim }}(\infty)}
$$

L'équation suppose une relation linéaire entre le degré d'hydratation et le retrait chimique des pâtes de ciment (Knudsen et Geiker, 1982 ; Parrot et al., 1990 ; GarciaBoivin, 2001 ; Mounanga et al., 2004a). Cependant, Knudsen et Geiker et Mounanga et al. ont mis en évidence une diminution à moyen et à long termes $(\alpha \geq 50 \%)$ de la valeur de retrait chimique de l'ordre de $0.5 \mathrm{~mm}^{3} \mathrm{~g}^{-1}{ }^{\circ} \mathrm{C}^{-1}$ (Knudsen et Geiker, 1982 ) à $0.8 \mathrm{~mm}^{3} \mathrm{~g}^{-1}{ }^{\circ} \mathrm{C}^{-1}$ (Mounanga et al., 2006), lorsque la température dépasse $40^{\circ} \mathrm{C}$. Cet effet de la température s'explique probablement par une modification de la morphologie des hydrates formés à des températures relativement élevées (Regourd et Gautier, 1980 ; Kjellsen et al., 1991). Regourd et Gautier ont en effet montré que jusqu'à $40^{\circ} \mathrm{C}$, les hydrates sont de même nature que ceux formés à $20^{\circ} \mathrm{C}$. Au-delà de $40^{\circ} \mathrm{C}$, des différences apparaissent dans la morphologie des silicates hydratés. Ils se développent en fibres longues plus individualisées qu'à $20^{\circ} \mathrm{C}$. Kjellsen et al. ont observé, sur des mortiers conservés en conditions endogènes à 5,20 et $50^{\circ} \mathrm{C}$, que la morphologie des cristaux de portlandite dépendait fortement de la température de conservation : de forme généralement lamellaire et allongée à faible température, ils se présentent sous un aspect beaucoup plus compact lorsque la température est plus élevée. 
Dans la suite de l'étude, nous nous limiterons au très jeune âge $(\alpha<50 \%)$ afin d’éviter l’influence de tels phénomènes sur le calcul du degré d’hydratation.

La figure 1 présente les évolutions de $E_{a}$ et de $\tilde{A} / \tilde{A}_{\max }$ en fonction de la maturité calculée à partir de la loi d'Arrhenius (Wirquin et al., 2002), pour des pâtes de ciment de rapport $E / C$ égal à 0,25 et 0,40 et à différentes températures $T$ de cure. Les résultats montrent une évolution rapide de l'énergie d'activation, dépendant de la température dès les premières heures puis une stabilisation au cours du temps sur 24h d'hydratation. Cette forme d'évolution peut être rapprochée de celles présentées par d'Aloïa et Chanvillard (d'Aloïa, 1998 ; d'Aloïa et Chanvillard, 2002).
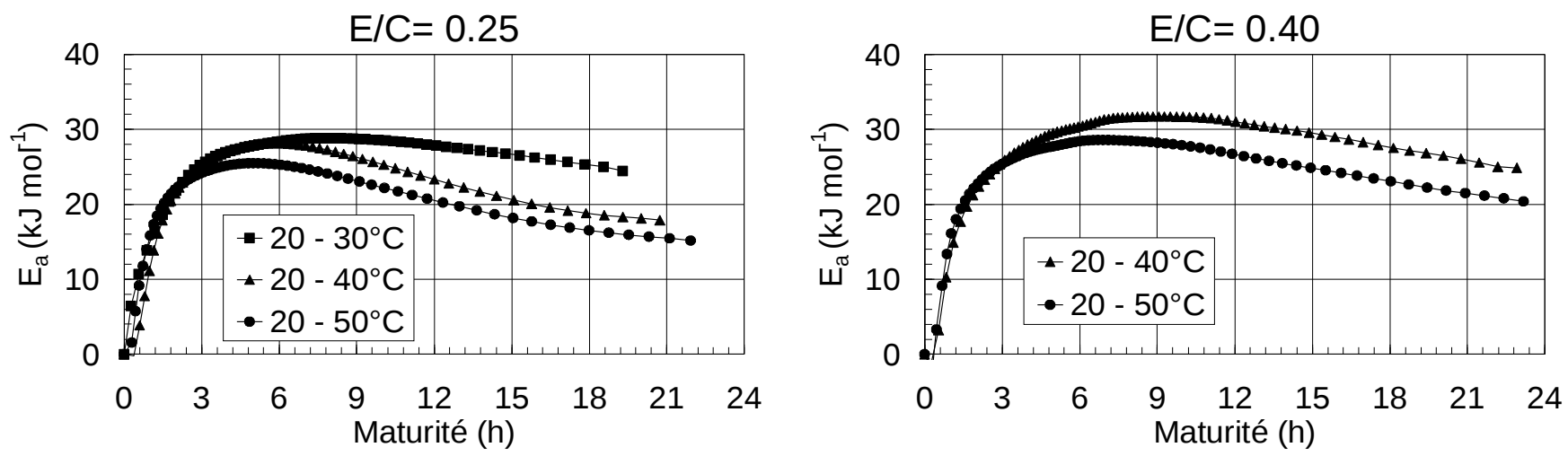

Energie d'activation apparente
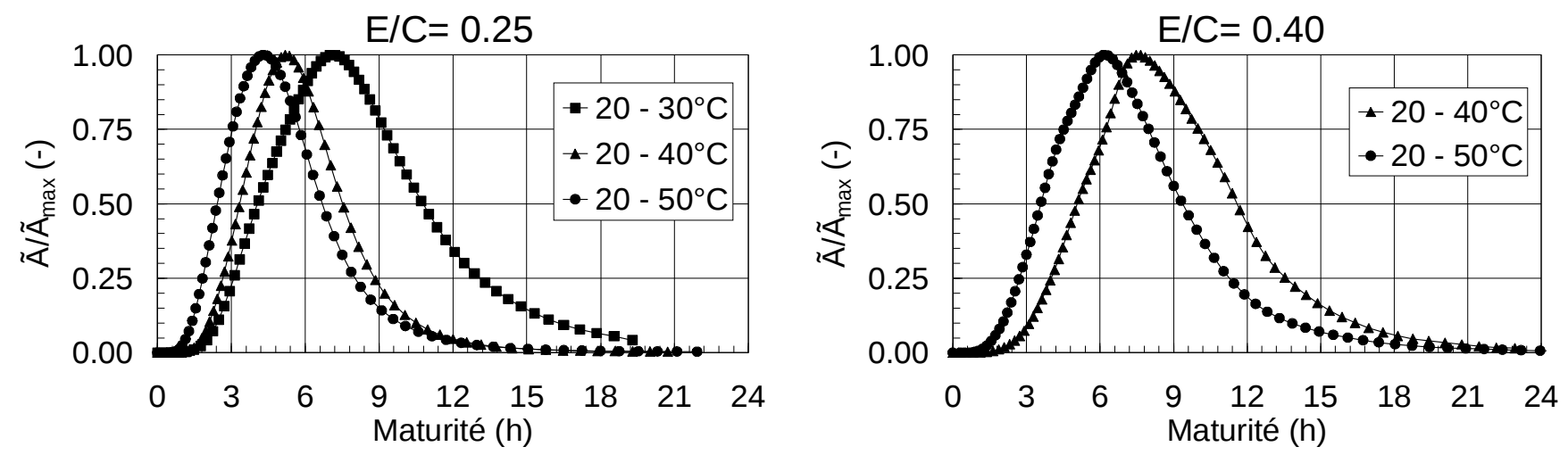

Affinité chimique normée
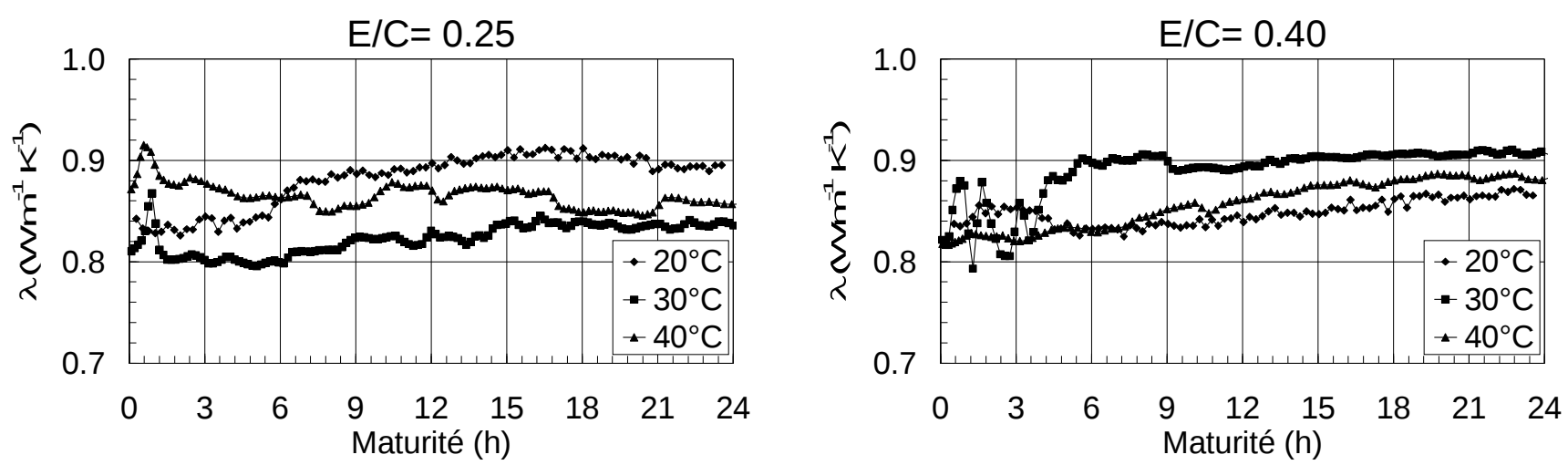

Conductivité thermique 

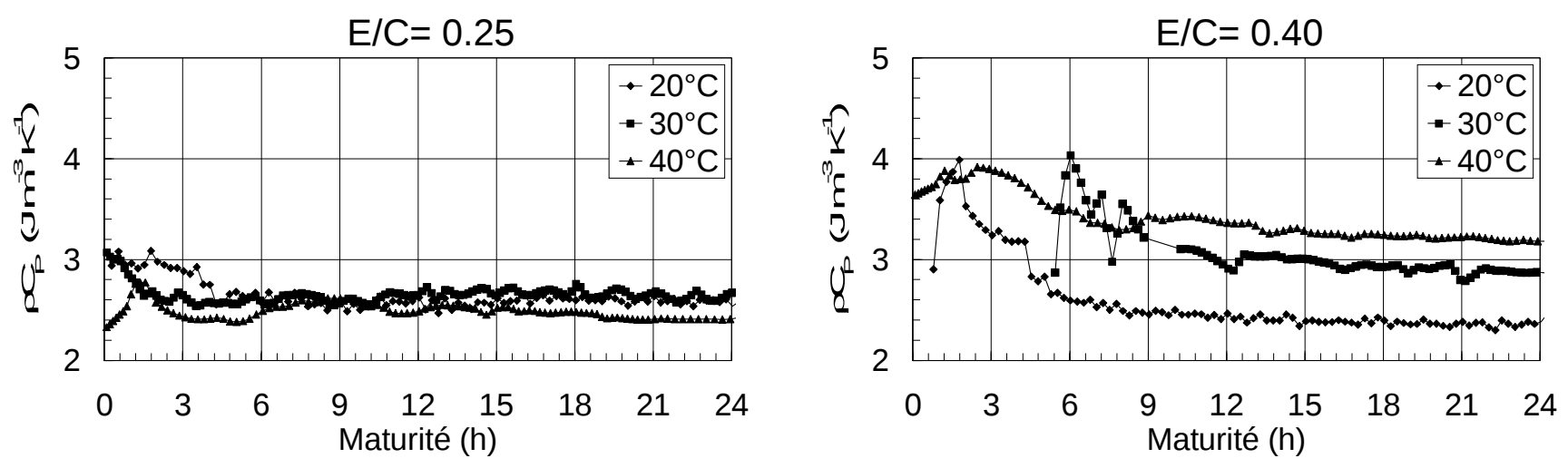

Capacité calorifique volumique

Figure 1. Résultats expérimentaux

\subsection{Propriétés thermophysiques : $\lambda$ et $\rho C_{P}$}

$\lambda$ et $\rho C_{P}$ désignent, respectivement, la conductivité thermique et la capacité calorifique volumique des matrices cimentaires. $\lambda$ mesure la capacité du matériau à transférer la chaleur tandis que $\rho C_{P}$ correspond à la quantité d'énergie nécessaire pour élever la température d'un milieu homogène d' $1 \mathrm{~K}$. On pourra trouver dans (Bentz, 2006) une revue bibliographique récente sur les propriétés thermiques des pâtes de ciment au jeune âge. Dans cette étude, $\lambda$ et $\rho C_{P}$ ont été mesurées simultanément sur une même éprouvette en utilisant une méthode d'essai inspirée de la méthode des «fils chauffé et non chauffé » (Poullain et al., 2006). Pendant les essais, les éprouvettes sont maintenues à température quasi-uniforme $( \pm 1 \mathrm{~K})$. Les résultats sont présentés à la figure 1, en fonction de la maturité des pâtes de ciment.

On note une augmentation de la conductivité thermique au cours des premières 24 heures, liée à l'accroissement du volume occupé par la phase solide tandis que la capacité calorifique présente une évolution décroissante due à la consommation de l'eau libre par l'hydratation.

\section{Modélisation du couplage thermochimique au très jeune âge}

\subsection{Présentation du modèle thermochimique}

Le modèle mis au point permet la détermination de l'évolution du degré d'hydratation et du champ de température de matrices cimentaires aux jeune et très jeune âges. Il est utilisé ici pour quantifier l'influence des paramètres de transfert thermique sur l'évolution thermique et physico-chimique des pâtes de ciment.

Le principe de résolution, ainsi que les paramètres d'entrée et de sortie et les lois de comportement utilisées, sont présentés à la figure 2. Le milieu 2D modélisé est 
caractérisé par les dimensions de ses côtés $a$ et $b$, ajustables selon le type de pièces ou de structures étudiées. Les flux thermiques dans la troisième direction sont considérés nuls.

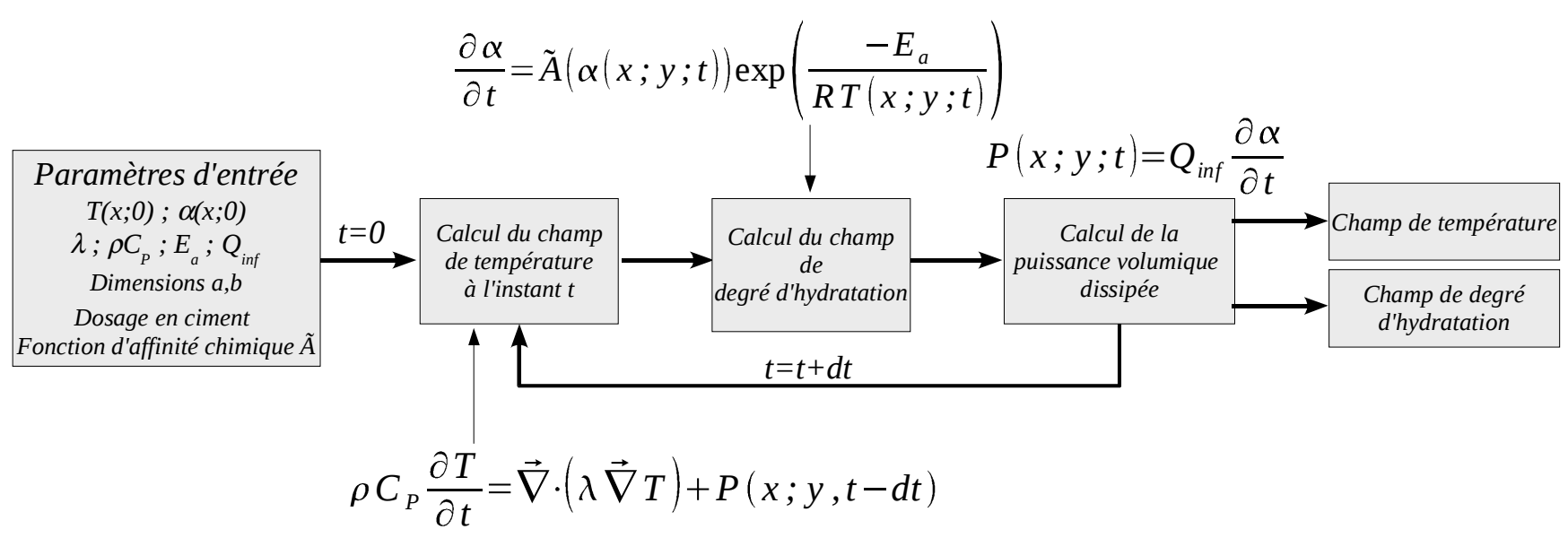

Figure 2. Organigramme du modèle thermochimique

Les calculs effectués prennent en compte un certain nombre d'hypothèses dont voici les principales :

$i \quad$ Milieu étudié en conditions endogènes,

ii Avancement global et uniforme des réactions d'hydratation des différentes phases du ciment, quantifié par le degré d'hydratation $\alpha(t)$,

iii Effet purement cinétique de la température sur le processus d'hydratation,

iv $E_{a}, \lambda$ et $\rho C_{P}$ constants au cours de l'hydratation,

$v \quad \tilde{A}(t)$ définie comme une fonction de $\alpha(t)$,

vi Transferts de chaleur par rayonnement et convection négligés.

Compte tenu des résultats expérimentaux présentés à la figure 1, certaines hypothèses (notamment iii et $i v$ ) peuvent paraître restrictives. Une version améliorée de la modélisation, prenant en compte des paramètres évolutifs en fonction du temps et de la température, est actuellement en cours de développement. Le modèle actuel est cependant suffisant pour une étude de sensibilité du calcul de $\alpha(t)$ et $T(t)$ vis-à-vis des valeurs de $\lambda$ et $\rho C_{P}$ utilisées.

\subsection{Jeu de paramètres considérés}

Le modèle est appliqué à la simulation de l'évolution des champs d'hydratation et de température d'une pâte de ciment de type CPA CEM I avec un rapport $E / C$ $=0,40$. Les résultats présentés ont été obtenus en imposant une température uniforme et constante de $20^{\circ} \mathrm{C}$ aux frontières de l'élément modélisé. Ces conditions aux 
frontières de type Dirichlet ( $1^{\text {ère }}$ espèce), aisément réalisables en laboratoire, ont été préférées à des conditions de Fourier ( $3^{\text {ème }}$ espèce), pourtant plus représentatives des conditions de maturation d'un béton sur chantier. Rappelons que le but premier de ces calculs est moins de simuler la maturation d'un béton en conditions réalistes que de faire une étude de sensibilité sur l'influence des valeurs des propriétés thermophysiques sur l'évolution thermique de la matrice cimentaire. Des calculs ont montré, qu'en considérant des conditions de Fourier, avec un coefficient d'échange moyen $h=10 \mathrm{~W} \mathrm{~m}^{-2} \mathrm{~K}^{-1}$ et une température ambiante de $20^{\circ} \mathrm{C}$, la température à coeur atteint plus de $90^{\circ} \mathrm{C}$ pour un échantillon de $25 \mathrm{~cm}$ de côté. Ces élévations deviennent alors largement incompatibles avec l'hypothèse d'un effet purement cinétique de la température sur les mécanismes d'hydratation.

Le tableau 2 et la figure 3 présentent, respectivement, les paramètres et la fonction d'affinité chimique introduits dans le modèle. $T(x, 0)$ et $\alpha(x, 0)$ correspondent aux valeurs initiales des champs de température et de degré d'hydratation du milieu simulé. $\lambda_{\text {moy }}$ et $\rho C_{P \text { moy }}$ sont les valeurs moyennes des propriétés thermophysiques calculées sur $24 \mathrm{~h}$ d'hydratation à partir des essais réalisés sur pâtes de ciment (figure $1-E / C=0,40 ; T=40^{\circ} \mathrm{C}$ ).

L'affinité présentée à la figure 3 est déterminée en considérant une énergie d'activation constante égale à $31700 \mathrm{~J} \mathrm{~mol}^{-1}$. Cette valeur de $E_{a}$ correspond à la valeur maximale mesurée sur la courbe $20-40^{\circ} \mathrm{C}$ (figure $1-E / C=0,40$ ).

\section{Tableau 2. Paramètres d'entrée du modèle}

\begin{tabular}{|c|c|}
\hline Paramètres [unité $]$ & Valeurs numériques \\
\hline$T(x, 0)\left[{ }^{\circ} \mathrm{C}\right]$ & 20 \\
\hline$\alpha(x, 0)[-]$ & $5 \times 10^{-3}$ \\
\hline$a=b=L[\mathrm{~m}]$ & 0,25 \\
\hline$E_{a}\left[\mathrm{~J} \mathrm{~mol}^{-1}\right]$ & 31700 \\
\hline$\tilde{A}(\alpha)=A \cdot \alpha^{B}(1-\alpha)^{C}\left[\mathrm{~s}^{-1}\right]$ & $\mathrm{A}=121,24 \mathrm{~s}^{-1} ; \mathrm{B}=1,15 ; \mathrm{C}=8,99$ \\
\hline$\lambda_{\text {moy }}\left[\mathrm{W} \mathrm{m}^{-1} \mathrm{~K}^{-1}\right]$ & 0,87 \\
\hline$\rho C_{\text {Pmoy }}\left[\mathrm{J} \mathrm{m}^{-3} \mathrm{~K}^{-1}\right]$ & $2,56 \times 10^{6}$ \\
\hline$\Delta V_{\text {chim }}(\infty)^{*}\left[\mathrm{~mm}^{3} \mathrm{~g}^{-1}\right]$ & 68,4 \\
\hline$Q_{\text {inf }}{ }^{* *}\left[\mathrm{~J} \mathrm{~g}^{-1}\right]$ & 470 \\
\hline Dosage en ciment $\left[\mathrm{kg} \mathrm{m}^{-3}\right]$ & 1393 \\
\hline
\end{tabular}

*: calculé à partir des données fournies dans (Bentz et al., 2005)

** : calculé à partir des données fournies dans (Bentz, 2000) 


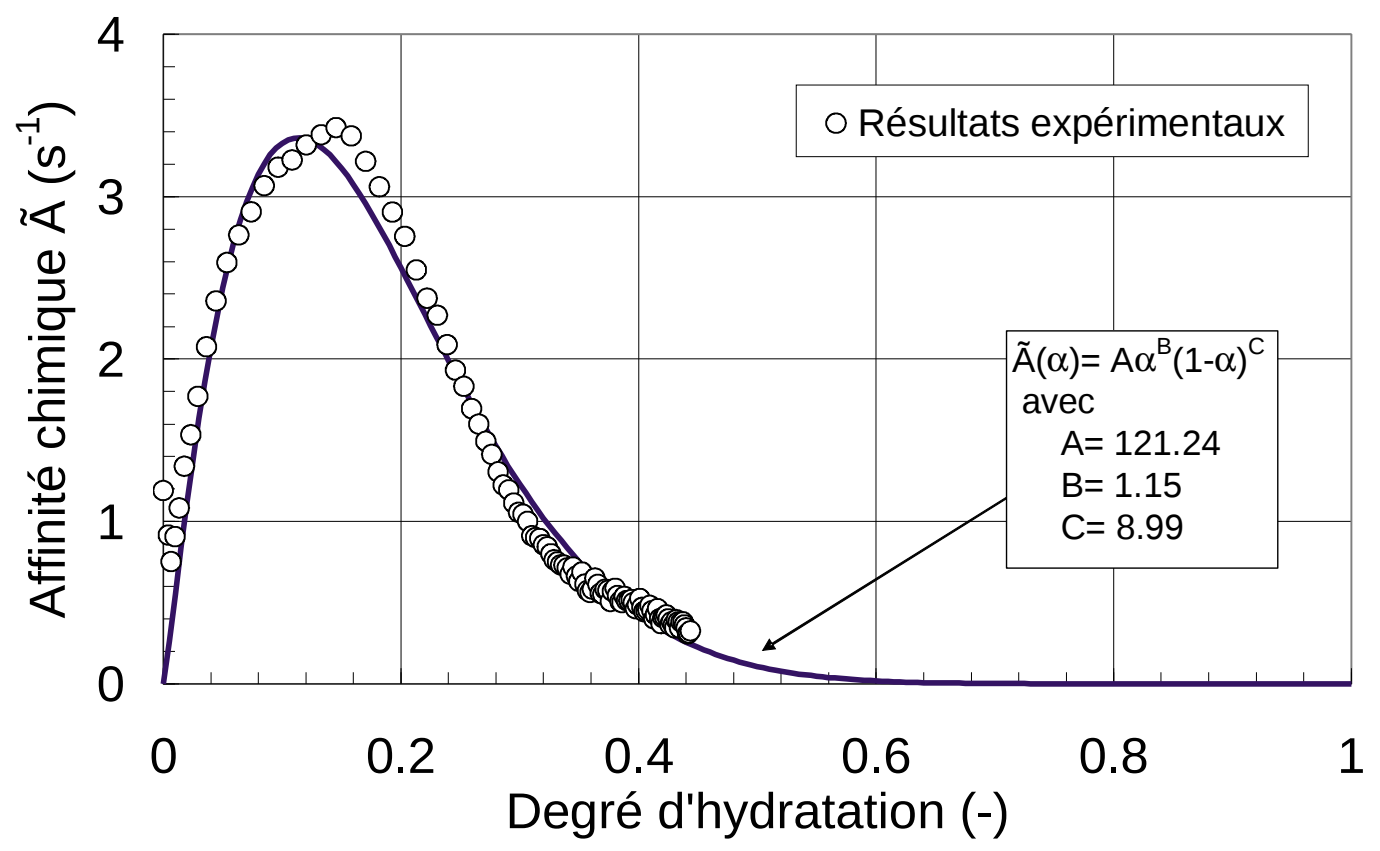

Figure 3. Fonction d'affinité chimique utilisée

\subsection{Résultats des simulations}

\subsubsection{Degré d'hydratation}

Afin de valider le modèle thermochimique présenté dans cet article, une comparaison entre les résultats donnés par le modèle du NIST (CEMHYD3D Bentz, 2000) et le modèle thermochimique et des mesures de degré d'hydratation par la méthode de la perte au feu $(\mathrm{PaF}$ - Bouasker et al., 2006) est présentée à la figure 4 .

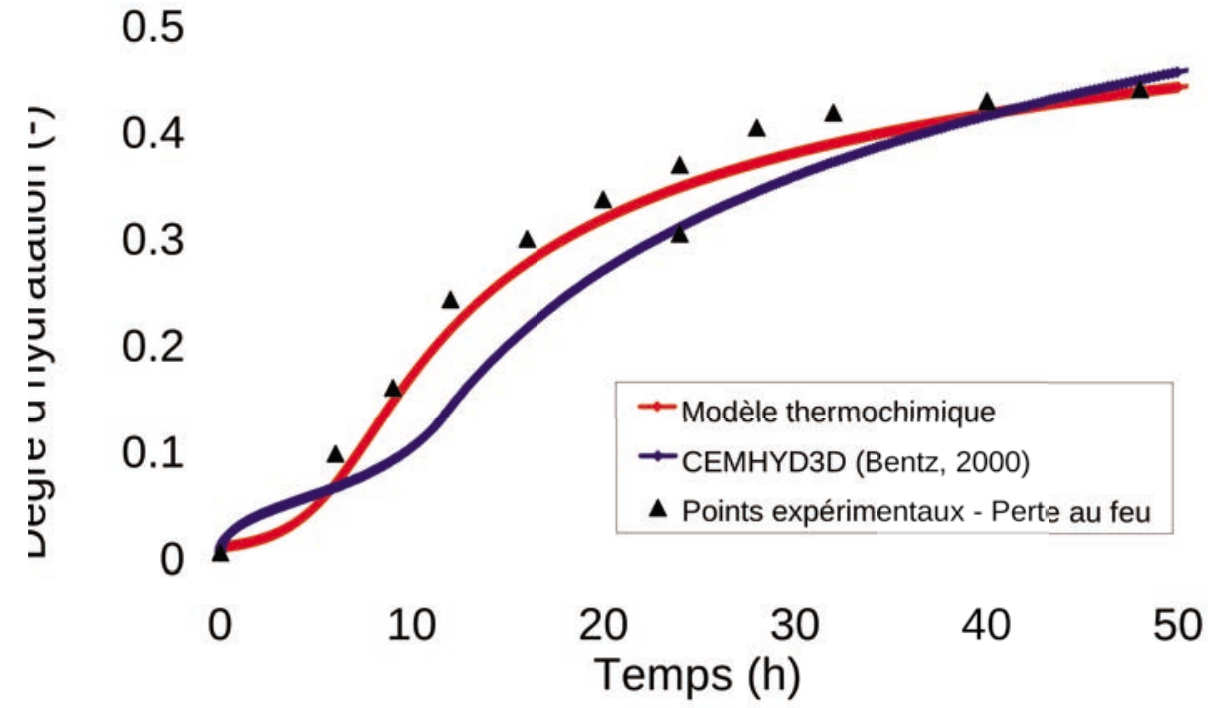

Figure 4. Comparaison entre modèles numériques et résultats expérimentaux obtenus sur pâtes de ciment à $\mathrm{E} / \mathrm{C}=0,40$ et $T=20^{\circ} \mathrm{C}$ 
On observe une bonne correspondance entre les deux modèles : l'écart absolu maximal entre les deux courbes demeure inférieur à 0,08. La concordance entre modèles numériques et résultats expérimentaux de $\mathrm{PaF}$ est également significative. Le modèle présenté permet donc de prédire correctement l'évolution du degré d'hydratation du ciment au très jeune âge, en conditions isothermes. Nous allons maintenant exploiter la capacité du modèle à simuler le couplage thermochimique du matériau.

Les simulations d'évolution du degré d'hydratation effectuées avec différentes valeurs de $\lambda$ et $\rho C_{P}$ sont représentées sur les figures 5 et 6 . On remarque une différence dans la cinétique d'hydratation et l'amplitude atteinte à $24 \mathrm{~h}$ d'hydratation entre le quart $(x=L / 4)$ et le centre $(x=L / 2)$ de la pièce. Cette différence, causée par l'élévation thermique plus importante au centre de l'éprouvette modélisée, est une conséquence de la thermoactivation de l'hydratation. On note par ailleurs une très faible influence de la variation de $\lambda$ et $\rho C_{P}$ sur la cinétique d'hydratation, dans la plage d'étude considérée (variation de $\pm 10 \%$ des propriétés thermiques et période considérée limitée à $24 \mathrm{~h}$ d'hydratation). Par exemple, à la figure 5 , la différence maximale entre les courbes reste inférieure à $2 \%$ d'hydratation.

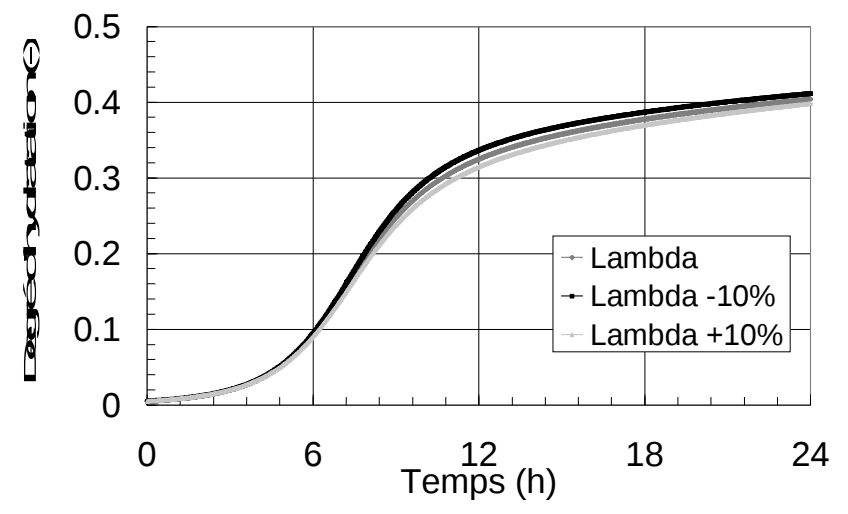

$x=L / 4$

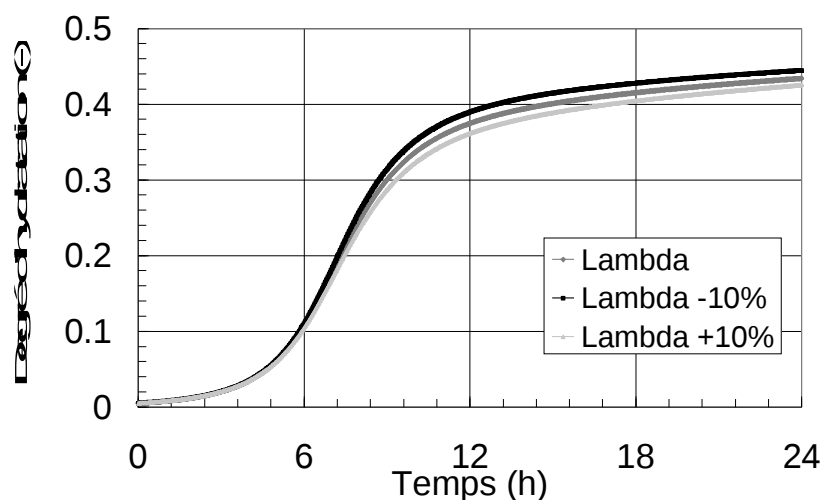

$x=L / 2$

Figure 5. Evolution du degré d'hydratation avec $\lambda_{\text {moy }} \pm 10 \%$ et $\rho C_{P \text { moy }}$
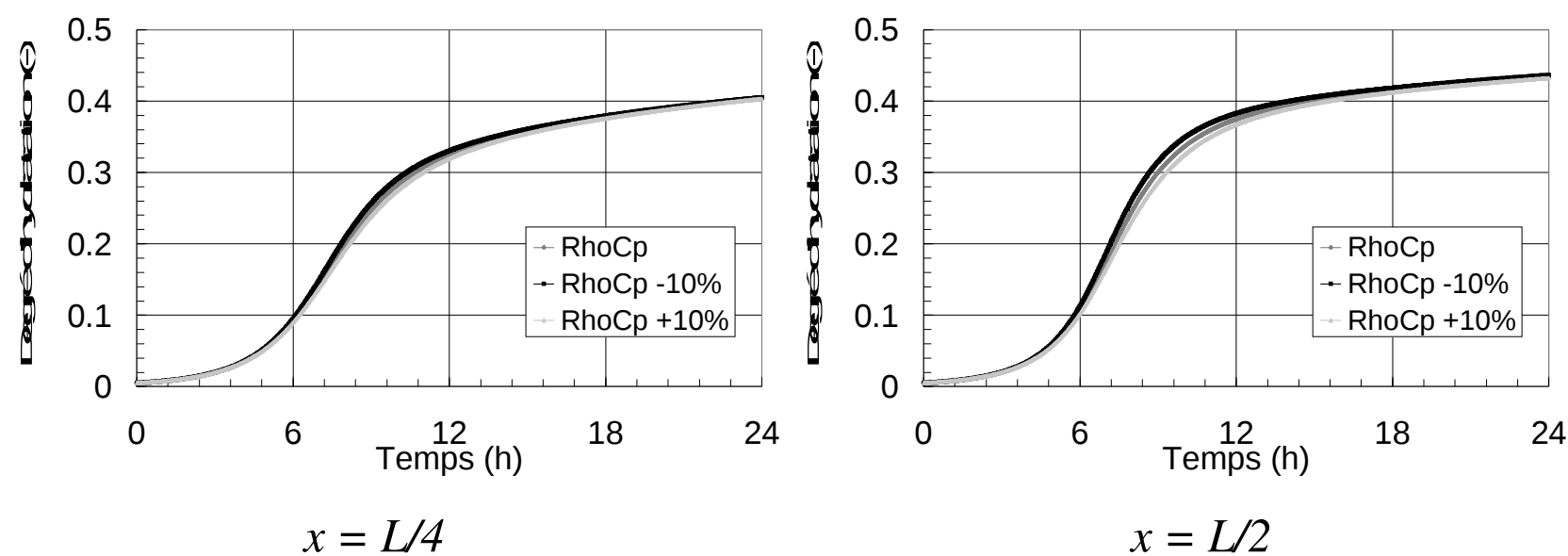

Figure 6. Evolution du degré d'hydratation avec $\rho C_{\text {Pmoy }} \pm 10 \%$ et $\lambda_{\text {moy }}$ 


\subsubsection{Champ de température}

Les champs de température calculés à partir du modèle développé sont présentés aux figures 7 et 8 . On observe un échauffement de la pièce lié à l'exothermie des réactions d'hydratation puis un refroidissement dû à la dissipation de chaleur dans le milieu ambiant, l'élévation en température étant plus faible lorsque l'on se rapproche des frontières de l'élément. L'influence des paramètres thermophysiques sur le champ de température est significative : elle se traduit, par exemple, à $x=L / 2$ au pic de température par une différence de $9,5 \mathrm{~K}$ entre les courbes $\lambda_{\text {moy }}-10 \%$ et $\lambda_{\text {moy }}+10 \%$ et de $5.5 \mathrm{~K}$ entre les courbes $\rho C_{P \text { moy }}-10 \%$ et $\rho C_{P \text { moy }}+10 \%$.

Ces résultats démontrent la sensibilité du calcul du champ de température vis-àvis du choix des paramètres thermophysiques : une prédiction correcte des variations thermiques d'un béton pendant sa prise et au-delà exige une détermination précise des propriétés thermiques du matériau au jeune âge.
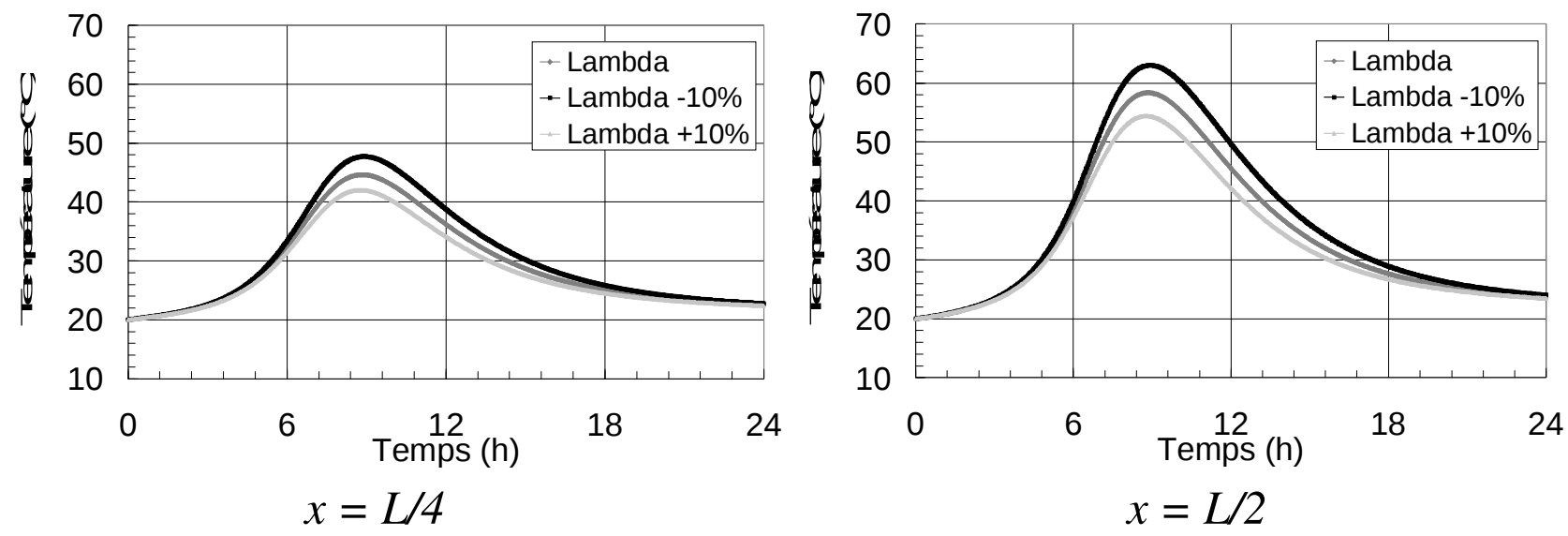

Figure 7. Evolution de la température avec $\lambda_{\text {moy }} \pm 10 \%$ et $\rho C_{\text {Pmoy }}$
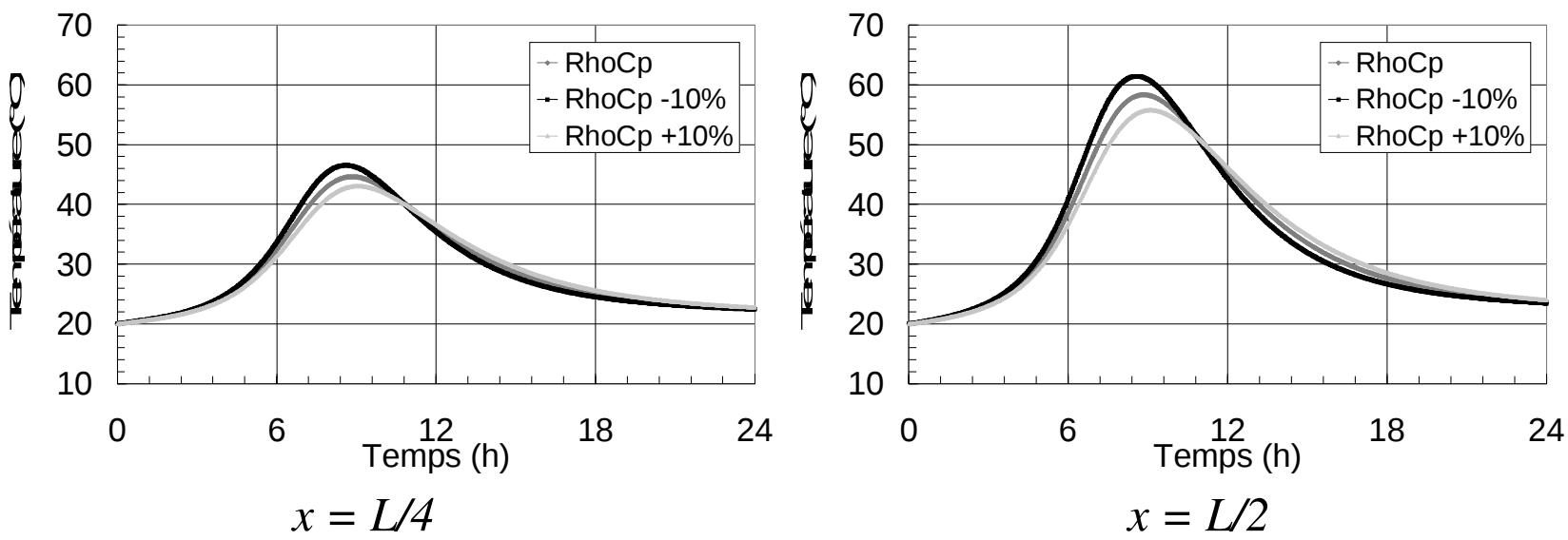

Figure 8. Evolution de la température avec $\rho C_{\text {Pmoy }} \pm 10 \%$ et $\lambda_{\text {moy }}$ 


\section{Conclusions et perspectives}

Les résultats expérimentaux présentés dans cet article ont mis en évidence les évolutions des propriétés thermophysiques et physico-chimiques de pâtes de ciment au très jeune âge. Ils constituent une base de données utile à une meilleure modélisation du comportement des matrices cimentaires durcissantes. Un modèle thermochimique a également été implémenté et a permis de mettre en évidence l'influence du choix des valeurs des propriétés de transfert thermique sur la simulation du comportement des matrices cimentaires au jeune âge : cette influence, très faible sur la cinétique d'hydratation du ciment, s'est révélée plus conséquente lors de la détermination du champ thermique du matériau. Une erreur sur la conductivité thermique entraîne une mésestimation de l'amplitude du pic de température tandis qu'une erreur sur la capacité calorifique aboutit à un décalage du pic de température dans le temps.

La présente modélisation ne concerne que des pâtes de ciment CEM I mais la méthode développée pourrait être adaptée à des compositions de liant intégrant des (poly)additions minérales (fumées de silice, laitiers de hauts fourneaux, cendres volantes notamment). L'étape préliminaire à cette adaptation est la détermination expérimentale de l'influence du dosage en additions minérales sur l'énergie d'activation apparente, l'affinité chimique et les propriétés thermophysiques du liant. Notons que les techniques expérimentales appliquées ici à la pâte de ciment pure peuvent être transposées à des pâtes adjuvantées (Mounanga et al., 2003) ou contenant des additions fines (Bouasker et al., 2005).

Les perspectives de ce travail sont le passage de la pâte de ciment au béton. Les granulats et les armatures peuvent être considérés comme inertes chimiquement, du moins au jeune âge du béton. Par contre, leur présence modifie sensiblement les propriétés thermophysiques du matériau (Torrenti et al., 1995 ; Morabito, 2001). En ce sens, les travaux de Bastian et Khelidj (Bastian et Khelidj, 1995) et de de Schutter et Taerwe (de Schutter et Taerwe, 1995) sur la mesure des propriétés thermophysiques des bétons au jeune âge constituent des voies de recherche possibles. Le calcul de ces paramètres à partir des propriétés de chaque phase (pâte de ciment, granulats) peut également être envisagé. Cependant, si l'estimation de la capacité calorifique effective du béton a été résolue grâce à l'additivité des enthalpies (Bentz et al., 1998 ; Waller, 2000), il n'en est pas de même pour la conductivité thermique équivalente, fortement dépendante de l'agencement des constituants et de la microstructure du milieu (Mounanga et al., 2004b). Des approches complémentaires, expérimentales et numériques, s'avèrent alors nécessaires. 
Cette étude a été en partie financée au travers du programme de recherche du LCPC « Durabilité, entretien, réparation et adaptation des infrastructures » (opération 1B021 : Durabilité du béton armé et de ses constituants : maîtrise et approche performantielle), piloté par Mme Véronique Baroghel-Bouny. Les auteurs sont reconnaissants du soutien apporté qui a permis la réalisation de ce travail de recherche. Les auteurs adressent également leurs remerciements à M. Marwen Bouasker pour la réalisation des mesures de degré d'hydratation par perte au feu.

\section{Bibliographie}

Acker P., Comportement mécanique du béton : Apports de l'approche physico-chimique, rapport de recherche $\mathrm{LPC}, \mathrm{n}^{\circ} 152,1988,121 \mathrm{p}$.

Acker P., Retraits et fissurations du béton. Documents scientifiques et techniques, Association Française Pour la Construction (AFPC), 1992, 42 p.

Bastian G., Khelidj A., «Propriétés thermophysiques d'un béton fraîchement coulé », Bulletin de liaison des LPC, vol. 200, 1995, p. 25-35.

Bentz D.P., Waller V., de Larrard F., "Prediction of adiabatic temperature rise in conventional and high-performance concretes using a 3-D microstructural model", Cement and Concrete Research, vol. 28, n², 1998, p. 285-297.

Bentz D.P., CEMHYD3D: A three-dimensional cement hydration and microstructure development modelling package. Version 2.0, rapport de recherche NISTIR 6485, avril 2000, NIST, U.S. Department of Commerce.

Bentz D.P., Lura P., Roberts J., "Mixture proportioning for internal curing", Concrete International, vol. 27, n², 2005, p. 35-40.

Bentz D.P., "Transient plane source measurements of the thermal properties of hydrating cement pastes", accepté pour publication dans Materials and Structures en 2006

Bernard O., Ulm F.-J., Lemarchand E., "A multiscale micromechanics-hydration model for the early-age elastic properties of cement-based materials", Cement and Concrete Research, vol. 33, n9, 2003, p. 1293-1309.

Bouasker M., Turcry P., Mounanga P., Loukili A., "Influence of limestone filler on chemical shrinkage and hydration of cement pastes at early age", In: Pijaudier-Cabot G., Gérard B., Acker P, éditeurs, Proceedings of the 7th International conference on creep, shrinkage and durability of concrete and concrete structures, Nantes, 12-14 septembre 2005, Paris, Hermès Science Publishing, p. 559-564.

Bouasker M., Mounanga P. ̃̃ , Turcry Ph., Loukili A., Khelidj A., "Chemical shrinkage of cement pastes and mortars at very early age: Effect of limestone filler and granular inclusions", soumis à Cement and Concrete Composites en 2006

D'Aloïa L., Détermination de l'énergie d'activation apparente du béton dans le cadre de l'application de la méthode du temps équivalent à la prévision au jeune âge : Approches expérimentales mécaniques et calorimétrique, simulations numériques, Thèse de doctorat, INSA de Lyon, 1998. 
D’Aloïa L., Chanvillard G., "Determining the "'apparent'” activation energy of concrete $\mathrm{E}_{\mathrm{a}}$ Numerical simulations of the heat of hydration of cement", Cement and Concrete Research, vol. 32, $\mathrm{n}^{\circ} 8,2002$, p. 1277-1289.

De Schutter G., Taerwe L., "Specific heat and thermal diffusivity of hardening concrete", Magazine of Concrete Research, vol. 47, n¹72, 1995, p. 203-208.

De Schutter G., "Finite element simulation of thermal cracking in massive hardening concrete elements using degree of hydration based material laws", Computers and Structures, vol. $80, \mathrm{n}^{\circ} 27-30,2002$, p. 2035-2042.

Garcia-Boivin S., Retrait au jeune âge du béton : Développement d'une méthode expérimentale et contribution à l' analyse physique du retrait endogène, Thèse de doctorat, ENPC (Paris), 2001.

Kjellsen K.O., Detwiler R., Gjørv O.E., "Development of microstructures in plain cement pastes hydrated at different temperatures”, Cement and Concrete Research, vol. 21, n¹, 1991, p. 179-189.

Knudsen T., Geiker M., "Chemical shrinkage as an indicator of the stage of hardening", Conférence internationale sur le béton au jeune âge, ENPC (Paris), vol. I, 1982, p. 163165.

Lackner R., Mang H.A., "Chemoplastic material model for the simulation of early-age cracking: From the constitutive law to numerical analyses of massive concrete structures", Cement and Concrete Composites, vol. 26, n5, 2002, p. 551-562.

Morabito P., Thermal properties of concrete: Variations with the temperature and during the hydration phase, rapport de recherche BE96-3843/2001:18-4, mai 2001, projet IPACS.

Mounanga P., Loukili A., Khelidj A., "Influence of superplasticizer on activation energy and autogenous shrinkage of a cement paste at early age", ACI Special Publication "7th CANMET/ACI International conference on superplasticizers and other chemical admixtures in concrete", $\mathrm{n}^{\circ} 217,2003, \mathrm{p}$. 513-528.

${ }^{a}$ Mounanga P., Khelidj A., Loukili A., Baroghel-Bouny V., "Predicting $\mathrm{Ca}(\mathrm{OH})_{2}$ content and chemical shrinkage of hydrating cement pastes using analytical approach", Cement and Concrete Research, vol. 34, n², 2004, p. 255-265.

${ }^{\mathrm{b}}$ Mounanga P., Khelidj A., Bastian G., "Experimental study and modelling approaches for the thermal conductivity evolution of hydrating cement paste", Advances in Cement Research, vol. 16, $\mathrm{n}^{\circ} 3,2004$, p. 95-103.

Mounanga P., Khelidj A., Baroghel-Bouny V., Loukili A., «Evolution physico-chimique et retrait endogène des matrices cimentaires au très jeune âge : Apports de l'expérimentation », 2èmes Journées «Durabilité »du Réseau des LPC, "Durabilité 2006 : Méthodes d'essais et applications », Paris, 15-16 mai 2006.

Parrott L.J., Geiker M., Gutteridge W.A., Killoh D. "Monitoring Portland cement hydration: Comparison of methods", Cement and Concrete Research, vol. 20, n6, 1990, p. 919-926.

Poullain Ph., Mounanga P., Bastian G., Coué R., "Determination of the thermophysical properties of evolutive porous media: application to Civil Engineering materials", The European Physical Journal - Applied Physics, vol. 33, n¹, 2006, p. 35-49. 
Regourd M., Gautier E., «Comportement des ciments soumis au durcissement accéléré », Annales de l'ITBTP, $\mathrm{n}^{\circ} 387,1980$, p. 83-96.

Torrenti J.M., Aristaghes P., Dombernovski K., Edon F., Guenot I., Monachon P., « La simulation des effets thermiques dans le béton au jeune âge : Exemples d'applications », Annales de l'ITBTP, $\mathrm{n}^{\circ}$ 535, série Béton 322, 1995, p. 3-27.

Ulm F.-J., Coussy O., "Couplings in early-age concrete: From material modelling to structural design”, Int. Journal of Solids and Structures, vol. 35, n³1-32, 1998, p. 4295-4311.

Waller V., Relations entre composition des bétons, exothermie en cours de prise et résistance en compression, Thèse de doctorat, ENPC (Paris), 2000.

Waller V., d'Aloïa L., Cussigh F., Lecrux S., "Using the maturity method in concrete cracking control at early ages", Cement and Concrete Composites, vol. 26, n5, 2004, p. 589-599.

Wirquin E., Broda M., Duthoit B., "Determination of the apparent activation energy of one concrete by calorimetric and mechanical means. Influence of a superplasticizer", Cement and Concrete Research, vol. 32, n8, 2002, p. 1207-1213. 